\title{
COVID-19 in pregnancy in South Africa: Tracking the epidemic and defining the natural history
}

South Africa (SA) has seen a rapid increase in COVID-19 infections in recent weeks, with cases exceeding 40000 in early June and anticipated to escalate rapidly as lockdown is eased. The country also has the largest HIV burden globally, and poor maternal and child health indices in many parts. Although early indications were that COVID-19 infection does not worsen pregnancy and birth outcomes, ${ }^{[1-4]}$ recent reports have raised fresh concerns. ${ }^{[5]}$ Preterm birth ${ }^{[6-8]}$ neonatal pneumonia ${ }^{[9-11]}$ and cases of vertical transmission and postpartum infections have been reported, including in SA. ${ }^{[1,11-15]}$ Some maternal deaths related to COVID-19 have occurred, possibly linked to haemodynamic changes immediately postpartum $^{[2,16]}$ and/or to the thrombogenic nature of both pregnancy and COVID19. ${ }^{[17-19]}$ Maternal wellbeing in pregnant women with COVID-19 infection is a major concern, as these women often have high anxiety about infecting their newborn child, and may experience challenging interactions with healthcare providers and community stigma. ${ }^{[2,18]}$ Most evidence on COVID-19 and pregnancy to date is limited to case series, involves only symptomatic women without HIV, and is almost exclusively from high-income countries. Cohort data across a range of settings and population groups are the only means of fully understanding the natural history, clinical disease spectrum and risks of COVID-19 in pregnant women, fetuses and infants. ${ }^{[19,20]}$

Pregnant women have multiple interactions with the health system, with important implications for SARS-CoV-2 transmission to and from health workers and other patients and to newborns. ${ }^{[21-23]}$ One study in New York, USA, for example, performed universal SARS-CoV-2 testing in all women in an obstetric ward. In total, $15 \%$ of the 221 women were SARS-CoV2 -positive, and $88 \%$ of those who were infected were asymptomatic. ${ }^{[24]}$ In another study in Connecticut, USA, $2.9 \%$ of $~ 750$ women tested at delivery were COVID-19positive and asymptomatic. ${ }^{[25]}$ It is clear that an infection control strategy and research agenda that includes asymptomatic pregnant women is necessary. ${ }^{[26]}$ This editorial describes a set of surveillance and research activities among pregnant women that could provide key evidence to inform the response to COVID-19 in SA.

\section{Sentinel surveillance} among pregnant women Sentinel surveillance of infectious disease among pregnant women is commonly used as an indicator of disease burden at a population level. This approach was used during the height of the HIV epidemic in sub-Saharan Africa and elsewhere to track the epidemic and derive population-based HIV prevalence estimates. ${ }^{[27-29]}$ With serial surveys in sentinel clinics one can track population-level incidence, quantify case detection rates and assess the relative effectiveness of different control measures. Identifying infections in pregnant women attending antenatal clinics may allow for timely identification and control of local clusters of infections ${ }^{[30]}$ and for evidence-based decisions around lockdown deconfinement in different locales. ${ }^{[31-33]}$ Alternative surveillance methods such as household surveys are logistically challenging, costly, often have high refusal rates, have a low yield, and are unable to give local estimates unless sample sizes are considerable. ${ }^{[34-36]}$

COVID-19 surveillance in antenatal clinics may be particularly important in heavily affected provinces and in areas that are densely populated. These include informal settlements and areas with predominantly high-rise apartment buildings, such as
Hillbrow in Johannesburg, ${ }^{[37]}$ which may be vulnerable to the kind of outbreaks that occurred in New York City. Similarly, surveillance in SA should prioritise economic hubs with overcrowded informal densely populated settlements that may have poor access to health services and cover a range of geographical areas, including metropolitan hubs in all provinces. Surveillance could neatly be layered onto existing initiatives and collaborations, such as the recently launched hospitalfocused Maternal and Perinatal Outcomes of Novel Coronavirus in Pregnancy Study and a national pregnancy exposure registry, planned for implementation in 19 facilities in Gauteng, KwaZulu-Natal and Western Cape provinces. ${ }^{[38]}$ Establishing sentinel site pregnancy exposure registries, harmonised across sites and consolidating routine clinical data on pregnancy exposures and outcomes, provides a platform onto which additional surveillance activities such as with COVID19 can be built as the need arises.

Surveillance activities would need to adopt innovative approaches to COVID-19 testing given the limited availability of testing at present. Pregnant women with symptoms suggestive of COVID-19 would be prioritised for individualised polymerase chain reaction (PCR) testing of nasopharyngeal swabs,

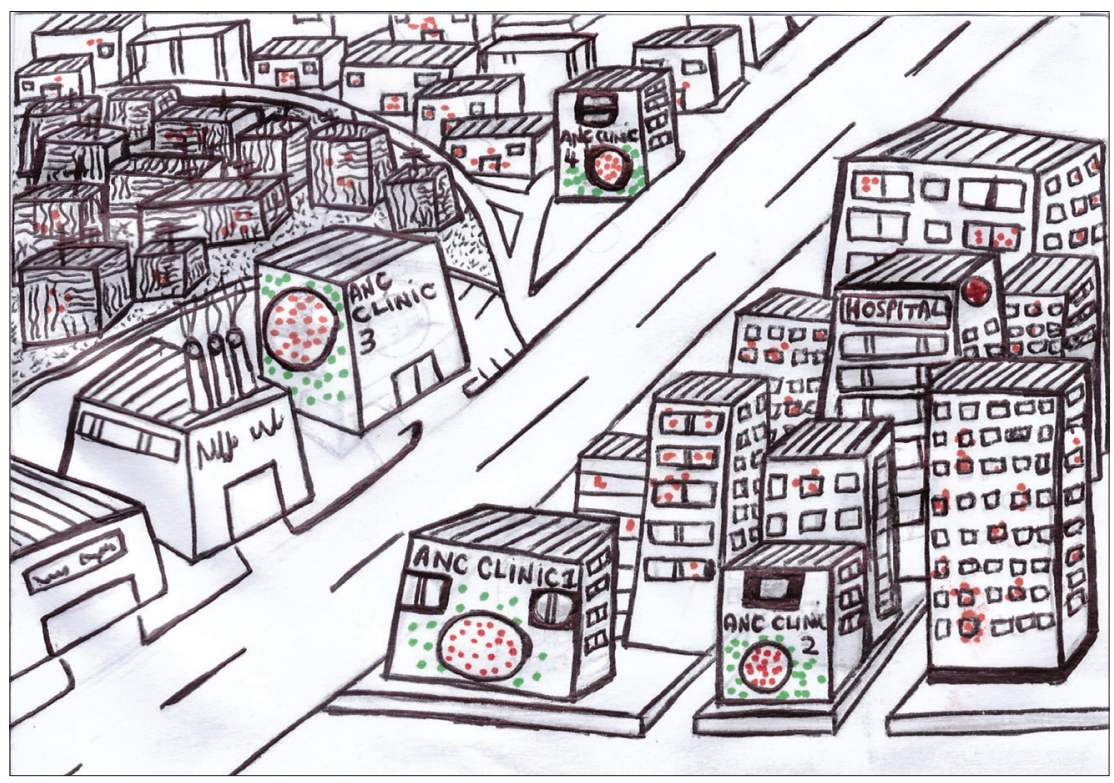

Proposed sentinel site surveillance of COVID-19 in pregnant women. The figure represents diverse environments including high-rise buildings, informal settlements, RDP (government subsidy) housing and the potential for pregnant women to represent COVID-19 infection within the broader population. (Red dots = COVID-19 infection; green dots = COVID-19-uninfected; ANC clinic = antenatal clinic.) 
as per national guidelines. Pooled testing among asymptomatic pregnant women may be an efficient alternative for diagnosis of acute infection and is increasingly being used in SA and elsewhere. ${ }^{[39-41]}$ This approach appears cost-effective up to a prevalence of SARSCoV-2 of $\sim 10 \% .{ }^{[39]}$ Nasopharyngeal or serological samples of $\sim 10$ women could be 'pooled' into one specimen. If that tests negative, it can be assumed that all 10 samples are negative. If the pooled test is positive, each sample in the pool would then be tested individually to detect the positive case(s). It is anticipated that serological testing will become available shortly and form the mainstay of active surveillance, and until then samples could be stored for later testing. In time, once barriers to COVID-19 testing have been addressed, 'universal testing' of pregnant women, a cornerstone of HIV programmes, may become a key element in COVID-19 control. Since antenatal cohorts represent diverse populations of women, testing of blood routinely collected may also provide opportunity for COVID-19 and other disease screening and testing.

Ideally, serological testing would be performed on serial maternal specimens, cord blood and infant blood to determine immune dynamics, which would have implications both for population seroprevalence and for informing the potential impact of future vaccination strategies. Although widespread sampling falls outside routine practice in public sector health services, practical approaches may be required, including limiting maternal sampling to certain time points, such as delivery, to optimally leverage routine blood tests such as maternal HIV testing and HIV viral load testing at delivery, infant birth HIV-PCR and stored dry blood spots.

\section{Cohorts of pregnant women with COVID-19}

Surveillance in sentinel antenatal clinics offers a unique opportunity to establish population-based prospective cohorts of women and children, which would be the optimal means of defining key COVID19 epidemiological parameters such as asymptomatic infection rates and case severity. ${ }^{[20]}$ These cohorts can also identify signals of adverse maternal or infant outcomes following COVID-19 infection. ${ }^{[42]}$ Natural history and birth outcomes could be disaggregated by HIV status, and between women living with HIV who initiate antiretroviral therapy in pregnancy and those on long-term treatment. In this cohort, ideally PCR sampling and serological sampling would be performed serially to determine immune dynamics and antibody kinesis. Here again, superimposing SARS-CoV-2-specific investigations on existing surveillance infrastructure that includes all pregnant women at a facility (such as the National Pregnancy Exposure Registry) could conserve resources and provide appropriate denominators for calculations. These platforms provide an opportunity for testing the performance of health interventions targeting COVID-19 such as therapeutics and vaccines in this important patient group. Then, once these modalities become more widely available, these systems serve to assess the safety of these products post-marketing in pregnant and postpartum women and their infants.

\section{Conclusions}

In summary, surveillance and cohort studies among pregnant and postnatal women and their infants could provide critical information. This includes estimates of COVID-19 infection rates in pregnant women, which are likely to be reflective of the disease burden in the broader community; an understanding of the natural history of COVID-19 in pregnant and postnatal women and their infants; and an assessment of innovative testing strategies such as pooled PCR and serological testing. Additionally, such data could determine whether COVID-19 disease manifests differently in women living with and without HIV infection. Lastly, this approach would provide a platform to evaluate whether or not pregnant women should be prioritised to be included in COVID-19 therapeutic and vaccine studies because of enhanced concerns about maternal and infant wellbeing. The considerable experience in SA of HIV research and prevalence surveys among pregnant women, together with the pregnancy registry infrastructure being developed, set the scene for a robust COVID-19 surveillance system and nested cohort studies in antenatal clinics.

\section{Lee Fairlie, Shobna Sawry, Faeezah Patel}

Wits Reproductive Health and HIV Institute, Faculty of Health Sciences, University of the Witwatersrand, Johannesburg, South Africa

\section{Jennifer E Balkus}

Department of Epidemiology, School of Public Health, University of Washington, Seattle, USA

\section{Emma Kalk}

Centre for Infectious Disease Epidemiology and Research, School of Public Health and Family Medicine, Faculty of Health Sciences, University of Cape Town, South Africa

\section{Portia Mutevedzi}

Respiratory and Meningeal Pathogens Research Unit, South African Medical Research Council, Johannesburg, South Africa; and School of Public Health, Faculty of Health Sciences, University of the Witwatersrand, Johannesburg, South Africa

\section{Karl-Günter Technau}

Empilweni Services and Research Unit, Rahima Moosa Mother and Child Hospital, Johannesburg, South Africa; and Department of Paediatrics and Child Health, Faculty of Health Sciences, University of the Witwatersrand, Johannesburg, South Africa

\section{Laura M Yates}

KwaZulu-Natal Research Innovation and Sequencing Platform (KRISP), School of Laboratory Medicine and Medical Sciences, University of KwaZulu-Natal, Durban, South Africa; and Department of Human Genetics, Inkosi Albert Luthuli Central Hospital, Durban, South Africa

\section{Amy Slogrove}

Department of Paediatrics and Child Health, Faculty of Medicine and Health Sciences, Stellenbosch University, Cape Town, South Africa

\section{Daynia Ballot}

School of Clinical Medicine, Faculty of Health Sciences, University of the Witwatersrand, Johannesburg, South Africa

\section{Rosella M Bandini}

Department of Paediatrics and Child Health, Faculty of Health Sciences, University of the Witwatersrand, and Charlotte Maxeke Johannesburg Academic Hospital, Johannesburg, South Africa

\section{Ushma Mehta}

Centre for Infectious Disease Epidemiology and Research, School of Public Health and Family Medicine, Faculty of Health Sciences, University of Cape Town, South Africa

\section{Dhayendre Moodley}

Department of Obstetrics and Gynaecology, School of Clinical Medicine, Faculty of Health Sciences, University of KwaZulu-Natal, Durban, South Africa 


\section{Ottancia Mhlongo}

HAST Unit, Strategic Health Programmes, KwaZulu-Natal

Department of Health, South Africa

\section{Samantha Budram}

Maternal-Fetal Medicine, Inkosi Albert Luthuli Central Hospital, Durban, South Africa; and University of KwaZulu-Natal, Durban, South Africa

\section{Salome Maswime}

Global Surgery Division, Department of Surgery, Faculty of Health Sciences, University of Cape Town, South Africa

\section{Valerie Vannevel}

SAMRC Maternal and Infant Health Care Strategies Unit, University of Pretoria, South Africa

\section{Helen Rees, Matthew Chersich}

Wits Reproductive Health and HIV Institute, Faculty of Health Sciences, University of the Witwatersrand, Johannesburg, South Africa mchersich@wrhi.ac.za

1. Chen $\mathrm{H}$, Guo J, Wang C, et al. Clinical characteristics and intrauterine vertical transmission potential of COVID-19 infection in nine pregnant women: A retrospective review of medical records. Lancet 2020;395(10226):809-815. https://doi.org/10.1016/S0140-6736(20)30360-3

2. Chen L, Li Q, Zheng D, et al. Clinical characteristics of pregnant women with Covid-19 in Wuhan, China. N Engl J Med 2020;385(25):e100. https://doi.org/10.1056/NEJMc2009226

3. Elshafeey F, Magdi R, Hindi N, et al. A systematic scoping review of COVID-19 during pregnancy and childbirth. Int J Gynaecol Obstet 2020;150(1):47-52 https://doi.org/10.1002/ijgo.13182

4. Ren H, Zhao L, Zhang A, et al. Early forecasting of the potential risk zones of COVID-19 in China's megacities. Sci Total Environ 2020;729:138995. https://doi.org/10.1016/j.scitotenv.2020.138995

5. Donders F, Lonnee-Hoffmann R, Tsiakalos A, et al. ISIDOG recommendations concerning COVID-19 and pregnancy. Diagnostics 2020;10(4):243. https://doi.org/10.3390/diagnostics10040243

6. Della Gatta AN, Rizzo R, Pilu G, et al. Coronavirus disease 2019 during pregnancy: A systematic review of reported cases. Am J Obstet Gynecol 2020;223(1):36-41. https://doi.org/10.1016/j.ajog.2020.04.013

7. Di Mascio D, Khalil A, Saccone G, et al. Outcome of coronavirus spectrum infections (SARS, MERS, COVID-19) during pregnancy: A systematic review and meta-analysis. Am J Obstet Gynecol MFM 2020;2(2 Suppl):100107. https://doi.org/10.1016/j.ajogmf.2020.100107

8. Yang Z, Wang M, Zhu Z, et al. Coronavirus disease 2019 (COVID-19) and pregnancy: A systematic review. J Matern Fetal Med 2020 (epub 30 April 2020). https://doi.org/10.1080/14767058.2020.17 59541

9. Baud D, Giannoni E, Pomar L, et al. COVID-19 in pregnant women: Authors' reply. Lancet Infect Dis 2020 (epub 17 March 2020). https://doi.org/10.1016/S1473-3099(20)30192-4

10. Khan S, Jun L, Nawsherwan, et al. Association of COVID-19 with pregnancy outcomes in health-care workers and general women. Clin Microbiol Infect 2020 (epub 8 April 2020). https://doi.org/10.1016/j. cmi.2020.03.034

11. Zimmermann P, Curtis N. COVID-19 in children, pregnancy and neonates: A review of epidemiologic and clinical features. Pediatr Infect Dis J 2020;39(6):469-477. https://doi.org/10.1097/ inf.0000000000002700

12. Cheruiyot I, Henry BM, Lippi G. Is there evidence of intra-uterine vertical transmission potential of COVID-19 infection in samples tested by quantitative RT-PCR? Eur J Obstet Gynecol Reprod Biol 2020;249:100-101. https://doi.org/10.1016/j.ejogrb.2020.04.034

13. Li D, Wang D, Dong J, et al. False-negative results of real-time reverse-transcriptase polymerase chain reaction for severe acute respiratory syndrome coronavirus 2: Role of deep-learning-based CT chain reaction for severe acute respiratory syndrome coronavirus 2: Role of deep-learning-based CT
diagnosis and insights from two cases. Korean J Radiol 2020;21(4):505-508. http://doi.org/10.3348/ diagnosis and

14. Kimberlin DW, Stagno S. Can SARS-CoV-2 infection be acquired in utero? More definitive evidence is needed. JAMA 2020;323(18):1788-1789. https://doi.org/10.1001/jama.2020.4868

15. Adebayo B. A two-day-old baby has died of Covid-19 in South Africa. CNN, 21 May 2020. https:// edition.cnn.com/2020/05/21/africa/south-africa-baby-death-coronavirus-int//index.html (accessed 29 June 2020).

16. Chen R, Zhang Y, Huang $\mathrm{L}$, et al. Safety and efficacy of different anesthetic regimens for parturients with COVID-19 undergoing cesarean delivery: A case series of 17 patients. Can J Anaesth 2020;67(6):655663. https://doi.org/10.1007/s12630-020-01630-7

17. Di Renzo GC, Giardina I. COVID-19 in pregnancy: Consider thromboembolic disorders and thromboprophylaxis. Am J Obstet Gynecol 2020;223(1):135. https://doi.org/10.1016/j.ajog.2020.04.017
18. Corbett GA, Milne SJ, Hehir MP, et al. Health anxiety and behavioural changes of pregnant women during the COVID-19 pandemic. Eur J Obstet Gynecol Reprod Biol 2020;249:96-97. https://doi. org/10.1016/j.ejogrb.2020.04.022

19. Breslin N, Baptiste C, Gyamfi-Bannerman C, et al. COVID-19 infection among asymptomatic and symptomatic pregnant women: Two weeks of confirmed presentations to an affiliated pair of New York City hospitals. Am J Obstet Gynecol MFM 2020;2(2 Suppl):00118. https://doi.org/10.1016/. ajogmf.2020.100118

20. Buekens P, Alger J, Breart G, et al. A call for action for COVID-19 surveillance and research during pregnancy. Lancet Glob Health 2020;8(7):e877-e878. https://doi.org/10.1016/S2214-109X(20)30206-0

21. Ashokka B, Loh MH, Tan CH, et al. Care of the pregnant woman with COVID-19 in labor and delivery: Anesthesia, emergency cesarean delivery differential diagnosis in the acutely ill parturient, care of the newborn, and protection of the healthcare personnel. Am J Obset Gynecol 2020;223(1):6674. https://doi.org/10.1016/.jajog.2020.04.005

22. Capanna F, Haydar A, McCarey C, et al. Preparing an obstetric unit in the heart of the epidemic strike of COVID-19: Quick reorganization tips. J Matern Fetal Med 2020 (epub 12 April 2020). https://doi org/10.1080/14767058.2020.1749258

23. Chawla D, Chirla D, Dalwai S, et al. Perinatal-neonatal management of COVID-19 infection Guidelines of the Federation of Obstetric and Gynecological Societies of India (FOGSI), Nationa Neonatology Forum of India (NNF), and Indian Academy of Pediatrics (IAP). Indian Pediat 2020;57(6):536-548

24. Sutton D, Fuchs K, D’Alton M, et al. Universal screening for SARS-CoV-2 in women admitted for delivery. N Engl J Med 2020;382(22):2163-2164. https://doi.org/10.1056/NEJMc2009316

25. Campbell KH, Tornatore JM, Lawrence KE, et al. Prevalence of SARS-CoV-2 among patients admitted for childbirth in southern Connecticut. JAMA 2020;323(24):2520-2522. https://doi.org/10.1001/ jama.2020.8904

26. Goudsmit J. The paramount importance of serological surveys of SARS-CoV-2 infection and immunity. Eur J Epidemiol 2020;35(4):331-333. https://doi.org/10.1007/s10654-020-00635-2

27. Sangal B, Kumar P, Dhingra N. HIV prevalence trend from HIV sentinel surveillance over a decade in India: An overview. Indian J Public Health 2018;62(2):138-142. https://doi.org/10.4103/ijph. IJPH_151_16

28. GBD 2016 Mortality Collaborators. Global, regional, and national under-5 mortality, adult mortality/ age-specific mortality, and life expectancy, 1970 - 2016: A systematic analysis for the Global Burden of Disease Study 2016. Lancet 2017;390(10100):1084-1150. https://doi.org/10.1016/S01406736(17)31833-0

29. Eaton JW, Rehle TM, Jooste S, et al. Recent HIV prevalence trends among pregnant women and all women in sub-Saharan Africa: Implications for HIV estimates. AIDS 2014;28(Suppl 4):S507-S514. https://doi.org/10.1097/QAD.0000000000000412

30. Thompson RN. Novel coronavirus outbreak in Wuhan, China, 2020: Intense surveillance is vital for preventing sustained transmission in new locations. J Clin Med 2020;9(2):498. https://doi.org/10.3390/ jcm9020498

31. Xiao SY, Wu Y, Liu H. Evolving status of the 2019 novel coronavirus infection: Proposal of conventional serologic assays for disease diagnosis and infection monitoring. J Med Virol 2020;92(5):464-467. https://doi.org/10.1002/jmv.25702

32. Anelli F, Leoni G, Monaco R, et al. Italian doctors call for protecting healthcare workers and boosting community surveillance during covid-19 outbreak. BMJ 2020;368:m1254. https://doi.org/10.1136 bmj.m1254

33. Dimeglio C, Loubes JM, Deporte B, et al. The SARS-CoV-2 seroprevalence is the key factor for deconfinement in France. J Infect 2020 (in press). https://doi.org/10.1016/j.jinf.2020.04.031

34. Gutreuter S, Igumbor E, Wabiri N, et al. Improving estimates of district HIV prevalence and burde in South Africa using small area estimation techniques. PLoS ONE 2019;14(2):e0212445. https://do org/10.1371/journal.pone.0212445

35. Kirakoya-Samadoulougou F, Nagot N, Samadoulougou S, et al. Declining HIV prevalence in parallel with safer sex behaviors in Burkina Faso: Evidence from surveillance and population-based surveys. Glob Health Sci Pract 2016;4(2):326-335. https://doi.org/10.9745/GHSP-D-16-00013

36. Marsh KA, Nyamukapa CA, Donnelly CA, et al. Monitoring trends in HIV prevalence among youn people, aged 15 to 24 years, in Manicaland, Zimbabwe. J Int AIDS Soc 2011;14(1):27. https://doi. org/10.1186/1758-2652-14-27

37. Rees H, Delany-Moretlwe S, Scorgie F, et al. At the heart of the problem: Health in Johannesburg' inner-city. BMC Public Health 2017;17(Suppl 3):554. https://doi.org/10.1186/s12889-017-4344-2

38. Mehta U, Kalk E, Fairlie L, et al. Why South Africa urgently needs to support the development of pregnancy exposure registries. S Afr Med J 2019;109(5):294-295. https://doi.org/10.7196/SAMJ.2019. v109i5.14008

39. Abdalhamid B, Bilder CR, McCutchen EL, et al. Assessment of specimen pooling to conserve SARS CoV-2 testing resources. Am J Clin Pathol 2020;153(6):715-718. https://doi.org/10.1093/ajcp/aqaa064

40. Eis-Hubinger AM, Honemann M, Wenzel II et al Ad hoc laboratory-based surveillance of SARSCoV-2 by real-time RT-PCR using minipools of RNA prepared from routine respiratory samples. I Clin Virol 2020;127:104381. https://doi.org/10.1016/j.jcv.2020.104381

41. Pouwels KB, Roope LSJ, Barnett A, et al. Group testing for SARS-CoV-2: Forward to the past? PharmacoEconomics Open 2020;4:207-210. https://doi.org/10.1007/s41669-020-00217-8

42. Alvarado MG, Schwartz DA. Zika virus infection in pregnancy, microcephaly, and maternal and feta health: What we think, what we know, and what we think we know. Arch Path Lab Med 2017;141(1):2632. https://doi.org/10.5858/arpa.2016-0382-RA

S Afr Med J 2020;110(9):728-731. https://doi.org/10.7196/SAMJ.2020.v110i9.15012 